AN. MED INTERNA (Madrid) Vol. 22, N. $^{\circ} 9$, pp. $445-448,2005$

\title{
Problemática de la deficiencia de yodo durante la gestación
}

\author{
D. A. DE LUIS, R. ALLER, O. IZAOLA \\ Instituto de Endocrinología y Nutrición. Facultad de Medicina. Unidad de Apoyo a la \\ Investigación. Hospital Universitario Río Hortega. Valladolid
}

IODINE DEFICIENCY DURING PREGNANCY

\section{RESUMEN}

El yodo es un micronutriente esencial para el cuerpo, que debe administrarse regularmente a través de la alimentación. Su función es intervenir en la síntesis de hormonas tiroideas. Las hormonas tiroideas intervienen en los procesos de desarrollo cerebral, del crecimiento y en la regulación del metabolismo. El déficit de yodo no solo se relaciona con el bocio, el problema es mucho más amplio, generando lo que se denomina -trastornos causados por deficiencia de yodo- (TYD), como son el aumento de mortalidad neonatal, defectos de audición y disminución de la capacidad intelectual y del crecimiento, aumento del número de abortos, anomalías congénitas con daño neuromotor permanente.

Uno de los grupos de riesgo, es la mujer gestante. Son diversos los trabajos que han mostrado la utilidad de la suplementación con yodo en este colectivo. Una revisión sistemática por el grupo colaborativo Cochrane, mostró como la suplementación con yodo durante la gestación disminuye la mortalidad infantil RR 0,71 (0,56-0,9). Además esta suplementación disminuyó la incidencia de cretinismo evaluado en niños menores de 4 años RR 0,27 (0,12-0,6), y mejora el desarrollo psicomotor.

Como recomendaciones finales podríamos decir que teniendo en cuenta que el yodo no tiene un pool de reserva corporal, se debería implantar un plan especial en gestantes similar a la suplementación con ácido fólico en el trimestre previo a la concepción y durante toda la gestación. Probablemente los preparados que presentan hierro y yodo sean superiores, a los de yodo solo (proporción $1 \mathrm{mg}$ de hierro por cada $25 \mu \mathrm{g}$ de yodo). Si la idea es un fármaco para embarazadas sería la presentación ideal. Teniendo en cuenta la incorporación de la mujer a la vida laboral y la existencia de horarios partidos, con toma de al menos una comida fuera del hogar, hace útiles los preparados en formas de tabletas. Las campañas de consumo de sal yodada han mostrado llegar solo al 50\% de la población.

PALABRAS CLAVE: Yodo. Deficiencia. Gestación.

\begin{abstract}
Iodine is an essential micronutrient, it would be administered every day with our diet. The main role of this micronutrient is the synthesis of thyroid hormone. Thyroid hormones are related with brain development and metabolic regulation. Iodine deficit is related with goitre, and an important problem "diseases related with iodine deficiency", including high rate of neonatal mortality, decrease of intelligence, delayed of growth, high rate of aborts and congenital abnormalities.

A risk group is pregnant women. Some authors have been demonstrated the utility of iodine supplementation during pregnancy. A systematic review of Cochrane group has shown that iodine supplementation during pregnancy decreased neonatal mortality $R R \quad 0.71$ (0.56-0.9), and decrease the incidence of cretinism in children under 4 years $R R \quad 0.27$ (0.12-0.6).

As final recommendations, a program in pregnant women must be development to treat with iodine such as we make with folic acid. Pills with iron and iodine (1 $\mathrm{mg}$ iron and $25 \mathrm{ug}$ iodine) have been demonstrated better results that pills with iodine. Tablets are the main presentation due to the role of the women in our Society and the work time. Programs of iodine enriched salt have been demonstrated a follow up of 50\%.
\end{abstract}

KEY WORDS: Yodine. Deficiency. Pregnancy.

De Luis DR, Aller R, Izaola O. Problemática de la deficiencia de yodo durante la gestación. An Med Interna (Madrid) 2005; 22: 445-448.

\section{INTRODUCCIÓN}

El yodo es un micronutriente esencial para el cuerpo, que debe administrarse regularmente a través de la alimentación.
Su función es intervenir en la síntesis de hormonas tiroideas. Las hormonas tiroideas intervienen en los procesos de desarrollo cerebral, del crecimiento y en la regulación del metabolismo. 
En 1992, bajo el auspicio de la OMS, el Comité Internacional para el Control de los Trastornos por Déficit de Yodo, La Asociación Europea de Tiroides, la Comisión de la Comunidad Europea y UNICEF fijaron, de manera consensuada, las siguientes recomendaciones (Tabla I). Después de numerosos estudios en áreas de pobre ingesta de yodo se recomienda durante el embarazo y la lactancia una dosis de yodo de 260 $\mu \mathrm{g} / \mathrm{dí}$ y así se está haciendo en varios países europeos (1-3).

\begin{tabular}{|c|c|}
\hline \multicolumn{2}{|c|}{ TABLA I } \\
\hline \multicolumn{2}{|c|}{ YODO EN $\mu \mathrm{g} / \mathrm{DI} A$} \\
\hline Niños prematuros & 90 \\
\hline Niños hasta 6 años & 90 \\
\hline Niños de 7 a 10 años & 120 \\
\hline Adultos & 150 \\
\hline Embarazadas y en período de lactancia & 200 \\
\hline
\end{tabular}

\section{FUENTES DIETÉTICAS DE YODO}

El yodo se encuentra distribuido de forma irregular en el agua y en los alimentos. Los mares son el mayor almacén de yodo; por tanto, el pescado de mar, es la principal fuente de yodo. El contenido en yodo de nuestro suelo es muy pobre, por lo que muchos de los productos agrícolas de zonas del interior y montañosas también son pobres en este elemento.

Además, el contenido en yodo de la leche y productos lácteos depende de la alimentación de los animales. Debido a ello y a nuestros hábitos alimentarios resulta difícil cubrir las necesidades diarias de yodo de la población a través de la dieta, y en la práctica esta deficiencia se evita suplementando la dieta con sal yodada. El yodo no presenta un pool de almacenamiento en el organismo, por lo que ha de ser repuesto continuamente. En la tabla II, mostramos diferentes fuentes artificiales de yodo. Teniendo en cuenta que no existe en la farmacopea española actual preparados de yoduro o yodato potásico (en forma de tabletas, gotas o grageas) adecuados para ello (4), hay que recurrir a preparados polivitamínicos y minerales que sí lo contienen, como Calcinatal ${ }^{\oplus}$, Multicentrum $^{\oplus}$, Superdyne ${ }^{\oplus}$ y Micebrina ${ }^{\circledast}$.

\section{DÉFICIT DE YODO, EPIDEMIOLOGÍA}

Hasta hace unos años el problema de la deficiencia de yodo se centraba en el bocio endémico, pero en las últimas décadas, las investigaciones llevadas a cabo en varios países han demostrado que el bocio no es la única manifestación de la carencia de yodo, sino también existen lo que podríamos denominar los trastornos causados por deficiencia de yodo (TYD), como el aumento de mortalidad neonatal, defectos de audición y disminución de la capacidad intelectual y del crecimiento, aumento del número de abortos, anomalías congénitas con daño neuromotor permanente. Todas estas anomalías inciden negativamente en la totalidad de la población condicionando un coeficiente de inteligencia inferior al de poblaciones similares sin deficiencia, y tienes graves repercusiones en el desarrollo socio económico de la comunidad.

\begin{tabular}{lr} 
& TABLA II \\
\hline Sal yodada & Contenido en yodo \\
Amiodarona & $60 \mathrm{ug} / \mathrm{g}$ \\
Desinfectantes & $7.500 \mathrm{ug} / \mathrm{comp}$ \\
\hline Solución de lugol & \\
Betadine & $126.000 \mathrm{ug} / \mathrm{ml}$ \\
Yoduro sodico al 10\% & $10.000 \mathrm{ug} / \mathrm{ml}$ \\
Vioformo/Clioquinol & $85.000 \mathrm{ug} / \mathrm{ml}$ \\
Enterovioformo & $12.000 \mathrm{ug} / \mathrm{ml}$ \\
Contrastes radiológicos & $120.000 \mathrm{ug} / \mathrm{ml}$ \\
\hline Hexabrix & \\
Oragrafin & $320.000 \mathrm{ug} / \mathrm{ml}$ \\
Lipiodol & $308.000 \mathrm{ug} / \mathrm{capsulas}$ \\
Renografin & $380.000 \mathrm{ug} / \mathrm{ml}$ \\
Telepaque & $370.000 \mathrm{ug} / \mathrm{ml}$ \\
\hline
\end{tabular}

La ingesta de yodo diaria recomendada en la mujer embarazada y lactante es de 200-300 ug/día. En una mujer embarazada teniendo en cuenta el efecto dilucional del mayor volumen urinario, la yoduria correspondería a $166 \mathrm{ug} / \mathrm{litro}$.

La magnitud del problema es enorme, de este modo la OMS (1990-1992) sugiere que 1.000 millones de personas en todo el mundo se encontraban en riesgo de padecer trastornos por yododeficiencia (TYD) al vivir en áreas donde el porcentaje de bocio en la población general era superior al 10\%. A medida que se disponen de más datos, las cifras van subiendo, estimandose en un informe de 1998, que la población en riesgo de padecer TYD se sitúa en 2.225 millones de personas, un $38 \%$ de la población general (Tabla III).

\begin{tabular}{lccc}
\multicolumn{4}{c}{ TABLA III } \\
& $\%$ Bocio & $\%$ Bocio & Variacion \% \\
\hline OMS & 1990 & 1998 & \\
Africa & 16 & 20 & +4 \\
America & 9 & 5 & -4 \\
Mediterraneos & 23 & 32 & +9 \\
Europa & 11 & 15 & +4 \\
Sudeste Asia & 13 & 12 & -1 \\
Pacifico Oeste & 9 & 8 & -1 \\
Total & 12 & 13 & +1 \\
\hline
\end{tabular}

Delange y cols. (6) ha resumido la situación actual en los países europeos, presentando en la Europa Central y del Oeste (32 estados), mostrando los siguientes datos; yododeficiencia (yoduria media < 100 ug/l) en 12 (Bélgica, Dinamarca, Fran- 
cia, Alemania, Grecia, Hungría, Irlanda, Italia, Rumanía, Eslovenia, España, Turquía, Yugoslavia), 14 estados suficientes en yodo (Austria, Bosnia, Bulgaria, Croacia, Chipre, Republica Checa, Finlandia, Macedonia, Holanda, Polonia, Portugal, Eslovaquia, Suiza, Reino Unido, Yugoslavia), de los 6 restantes con datos insuficientes parece que 4 serían yodosuficientes (Irlanda, Luxemburgo, Serbia y Noruega), y sin duda Albania y Montenegro continúan con yododeficiencia. En resumen 18 países con suficiente aporte de yodo y 14 persiste la yododeficiencia, suponiendo un $64 \%$ de los 600 millones de personas que viven en esta región.

Centrándonos en España, los diferentes trabajos existentes muestran, porcentajes de bocio en edad escolar (14-30\%), habiendose realizado estudios de campo en Cataluña, Galicia, Sevilla, Cádiz, Madrid, Las Hurdes, Huelva, Córdoba, Almería, Granada, Jaén, Málaga, Asturias, Navarra, León, País Vasco, Cuenca, Murcia, Guadalajara, Toledo, Teruel, Huesca y Zaragoza).

Más particularmente, en las mujeres gestantes, el grupo de la Dra. Morreale (7), ha realizado un trabajo en este colectivo en la Comunidad de Madrid. Se estudiaron un total de 419 gestantes sin suplementación y a 93 mujeres gestantes con suplementación (2 tabletas día de 150 ug IK). Por tanto, los resultados muestran que Madrid es una zona con déficit medio de yodo, los niveles de T41 de las mujeres embarazadas no alcanzan las cifras de mujeres con ingesta de yodo suplementada. Esta situación en el primer trimestre puede producir un riesgo de tener hijo con desarrollo psicomotor inferior. Se recomienda ingestas de 240-250 ug/día de yodo para conseguir yodurias de 160 a $166 \mathrm{ug} / \mathrm{l}$.

Otro estudio realizado en gestantes, mostró como existía una prevalencia de bocio del $52,6 \%$ y solo un $17 \%$ de las pacientes usaban sal yodada en el domicilio. La mediana de la yoduria fue de $113 \mathrm{ug} / \mathrm{L}$, con un $68 \%$ de las gestantes con una excreción < 150 ug/L (8).

MANIFESTACIONES CLÍNICAS DEL DÉFICIT DE YODO EN LA MADRE GESTANTE Y EN EL HIJO

Las poblaciones de riesgo incluyen a las mujeres embarazadas y en período de lactancia así como la población infantil, sobre todo en los primeros años de vida. Cuando el déficit de yodo afecta a la etapa fetal y al primer año de vida el daño producido es irreversible, ocasionando, según la intensidad de la carencia de yodo, disminución del coeficiente intelectual e incluso retraso mental.

Durante el embarazo y el período de lactancia están aumentadas las necesidades de yodo por lo que es de especial importancia recibir un aporte suficiente, ya que deben cubrirse las necesidades de yodo de la madre y a través de ella las del feto y del recién nacido. La carencia de yodo en la madre embarazada y en periodo de lactancia puede dar lugar a la aparición de bocio, también aumenta la tasa de hipotiroidismo y existe un mayor número de abortos y de muerte fetal.

A nivel fetal la carencia de yodo en el feto viene determinada por su carencia en la madre. Si el feto no dispone de suficiente yodo su tiroides puede aumentar de tamaño y ocasionar bocio que, aunque sea de pequeño volumen, en el momento del nacimiento puede provocar trastornos respiratorios y/o de la deglución. Además, la carencia de yodo ocasiona alteración de la función tiroidea, con retraso en el desarrollo del Sistema
Nervioso Central, así como del crecimiento y de la maduración ósea en mayor o menor grado dependiendo de la intensidad de la carencia. Cuando la carencia de yodo es grave hay riesgo de cretinismo bien de tipo neurológico bien de tipo mixedematoso (raros en nuestro país).

Una carencia de yodo en la madre en el periodo de lactancia también la padece el lactante, lo que contribuye a intensificar los trastornos del desarrollo físico y mental, secundarios a deficiencia de yodo durante la vida fetal.

\section{EXPERIENCIA DE LA SUPLEMENTACIÓN CON YODO}

La Organización Mundial de la Salud propugna la Yodación Universal de la sal, entendiéndose por ello la yodación de la sal de consumo humano y animal, incluyendo la utilizada en las industrias alimentarias. La primera experiencia de profilaxis de bocio endémico con yodo se inició en Ohio (USA) en 1917.

En España existe sal yodada disponible en el mercado desde 1983, Real Decreto de 27 de abril (1424/1983), que aprueba la reglamentación técnico-sanitaria para la obtención, circulación y venta de la sal yodada y establece el contenido de yodo en $60 \mathrm{mg}$ por $\mathrm{Kg}$ de sal, de tal manera que con la ingesta diaria normal de sal (unos 3 gramos/día) se cubren las necesidades diarias de yodo. En Galicia el 9 de enero de 1.985 se publicó en el DOGA un decreto sobre prevención del bocio endémico mediante el consumo de sal yodada. Actualmente en todos los comedores escolares de Galicia se utiliza sal yodada pero no existe garantía de su consumo en el hogar. En el año 2000 en las Cortes Generales se aprobó una proposición no de ley sobre (Yodación de sal para consumo humano y/o de animales) (22 de septiembre de 2000, serie D. No 61: 11-12).

Con el aporte adicional de yodo en cantidades fisiológicas (150-200 ug/dia) administrado a través de la sal yodada, no cabe esperar ningún riesgo para la salud ni suele presentarse intolerancia al yodo, tipo reacciones alérgicas. Una posible complicación de la profilaxis con sal yodada es la aparición de hipertiroidismo. Sin embargo los casos notificados fueron en su mayoría en personas de edad y atribuidos a la presencia de nódulos autónomos o a la enfermedad de Basedow-Graves subyacente.

Por tanto si nos planteamos realizar yodoprofilaxis debemos tener en cuenta estos apartados:

1. Utilización exclusiva de sal yodada para la preparación de la comida.

2. Debería utilizarse también sal yodada en la preparación de alimentos en la cadena de producción industrial (embutidos, pan, etc.).

3. Consumir frecuentemente, si es posible, pescado de mar, ya sea fresco, congelado o en conserva.

4. Si las medidas anteriores no son posibles se deberá administrar una suplementación diaria de yodo de 150-200 ug (IK) (presente en alguno de los complejos vitamínico y mineral existentes en el mercado).

Existen diversos autores que han evaluado algunos parámetros clínicos y bioquímicos al suplementar con yodo a mujeres embarazadas. De este modo, Pedersen y cols. (9), estudiaron 54 pacientes embarazadas suplementandolas con 200 ug de sal (IK) a un subgrupo, mostrando el grupo no tratado un aumento de TSH y volumen tiroideo durante el embara- 
zo. Estos datos habían sido ya corroborados en otro trabajo previo (10). En otro trabajo se mostró como el lipiodol $(0,5$ ml) por vía oral, disminuía la prematuridad y los abortos, con un aumento de la yoduria y la tiroxina (11). Algunos autores han comparado diferentes dosis, de este modo Antonangeli y cols. (12), compararon el efecto de $200 \mathrm{ug}$ de sal yodada (IK) frente a 50 ug de sal, siendo superior la primera dosis en cuanto a volumen tiroideo sin mostrar más tasa de tiroiditis postparto.

Recientemente se ha publicado una revisión sistemática por el grupo colaborativo Cochrane (13), mostrando como la suplementación con yodo durante la gestación disminuye la mortalidad infantil RR 0,71 (0,56-0,9), además esta suplementación disminuyó la incidencia de cretinismo evaluado en niños menores de 4 años RR 0,27 (0,12-0,6), así como mejor desarrollo psicomotor.

Por otra parte sería interesante conocer la utilidad de diferentes sales, no obstante no existen trabajos en gestantes. En población general, Zhao y cols. (14), realizaron un ensayo clínico con niños, para comparar la administración de un preparado de sal yodada (IK) (25 ppm), sal yodada del mercado y cápsulas de aceite yodado (400 mg). Mostrándose superior el preparado con $25 \mathrm{pm}$, sobre la sal de mercado. En cuanto a trabajos de titulación, en un trabajo se comparó en un ensayo clínico en población adulta, tres dosis de un preparado de sal yodada (IK), (200 mg, $400 \mathrm{mg}$ y $800 \mathrm{mg}$ ). Ninguna de las dosis superiores fueron más eficientes que $200 \mathrm{mg}$ (15). La biodisponibilidad de la sal yodada (3 cápsulas al día, $570 \mathrm{mg}$ por cápsula) frente a una dosis im de $1 \mathrm{ml}$ de lipiodol (480 mg) fue similar (16). Sin embargo, la biodisponibilidad puede variar al añadir otros elementos en el suplemento de yodo, de este modo Zimmermann y cols. (17) detectaron en niños como los comprimidos con (25 ug yodo potásico por g de sal y $1 \mathrm{mg}$ de hierro por $\mathrm{g}$ de sal) frente a la misma cantidad de sal solo de yodo, presentaron un mayor aumento de yoduria y volumen tiroideo.

\section{CONCLUSIONES}

La yododeficiencia es un problema real en Europa y más concretamente en España. No existe un Plan Nacional para la erradicación de los Trastornos por Deficiencia de Yodo, ni una legislación que regule el consumo de sal yodada a nivel nacional, solo las normas para yodar la sal.

Un grupo de alto riesgo son las mujeres gestantes, el déficit en el primer trimestre se traduce en alteraciones psicomotrices del recién nacido. A pesar de su trascendencia los trabajos de intervención son escasos y heterogeneos.

A pesar de ello podemos lanzar las siguientes recomendaciones:

1. Teniendo en cuenta que en el yodo no tiene un pool de reserva coporal, se debería implantar un plan especial gestantes similar a la suplementación con ácido fólico en el trimestre previo a la concepción y durante toda la gestación.

2. Probablemente los preparados que presentan hierro y yodo sean superiores, a los de yodo solo (proporción $1 \mathrm{mg}$ de hierro por cada 25 ug de yodo). Si la idea es un fármaco para embarazadas sería la presentación ideal.

3. Teniendo en cuenta la incorporación de la mujer a la vida laboral y la existencia de horarios partidos, con toma de al menos una comida fuera del hogar, hace útiles los preparados en formas de tabletas. Las campañas de consumo de sal yodada han mostrado llegar solo al $50 \%$ de la población.

\section{Bibliografía}

1. Escobar del Rey F, Morreale de Escobar G. Yodación universal de la sal: un derecho humano de la infancia. Endocrinología 1998; 45: 3-14.

2. Recommendations on iodine nutrition for mothers and infants in Europe, En: Delange F, Dunn JT, Glinoer D, editores. Iodine deficiency disorders in Europe: a continuing concern. Nueva York: Plenum Press, 1993; 471-8.

3. Escobar del Rey F, Morreale de Escobar G Yodación universal de la sal: un derecho humano de la infancia. Endocrinología 1998; 45: 4-16.

4. Arena Ansotegui J, Emparanza Knörr JI. Los antisépticos yodados no son inocuos. An Esp Pediatr 2000; 53: 25-9.

5. Morreale de Escobar G Interrelaciones materno-fetales de las hormonas tiroideas. An Esp Pediatr 1999; 50 (Supl. 125): 36-43.

6. Delange F, Dunn JT, Glinoer D Specific recommendation on iodine nutrition for Mothers and infants in Europe. En: Delange F, Dunn JT, Glinoer D, editores. Iodine Deficiency in Europe. Nueva York: Plenum Press, 1993; 478-9.

7. De Santiago J, Pastor I, Escobar del Rey F, Morreale de Escobar G. Thyroid function in pregnant women from an area with mild (grade I) iodine deficiency. J Endocrinol Inv 1999; 22 (Supl. 6): 68.

8. Abalovich M, Gutierrez S, Alcaraz G, Maccallini G, Garcia A, Levalle $\mathrm{O}$. Overt and subclinical hypothyroidism complicating pregnancy. Thyroid 2002; 12: 63-8.

9. Pedersen KM, Laurberg P, Iversen E, Knudsen PR, Gregersen HE, Rasmussen OS, et al. Amelioration of some pregnancy-associated variations in thyroid function by iodine supplementation. J Clin Endocrinol Metab 1993; 77: 1078-83.
10. Romano R, Jannini EA, Pepe M, Grimaldi A, Olivieri M, Spennati P, et al. The effects of iodoprophylaxis on thyroid size during pregnancy. Am J Obstet Gynecol 1991; 164: 482-5.

11. Chaouki ML, Benmiloud M. Prevention of iodine deficiency disorders by oral administration of lipiodol during pregnancy. Eur J Endocrinol 1994; 130: 547-51.

12. Antonangeli L, Maccherini D, Cavaliere R, Di Giulio C, Reinhardt B, Pinchera A, et al. Comparison of two different doses of iodide in the prevention of gestational goiter in marginal iodine deficiency: a longitudinal study. Eur J Endocrinol 2002; 147: 29-34.

13. Mahomed K, Gülmezoglu AM. Suplementos de yodo materno en areas deficitarias. Cochrane Database Syst Rev 2000: CD002837.

14. Zhao J, Xu F, Zhang Q, Shang L, Xu A, Gao Y, Chen Z, Sullivan KM, Maberly GF. Randomized clinical trial comparing different iodine interventions in school children. Public Health Nutr 1999: 173-8.

15. Elnagar B, Eltom M, Karlsson FA, Ermans AM, Gebre-Medhin M, Bourdoux PP. The effects of different doses of oral iodized oil on goiter size, urinary iodine, and thyroid-related hormones. J Clin Endocrinol Metab 1995; 80: 891-7.

16. Leverge R, Bergmann JF, Simoneau G, Tillet Y, Bonnemain B. Bioavailability of oral vs intramuscular iodinated oil (Lipiodol UF) in healthy subjects. J Endocrinol Invest 2003; 26 (Supl. 2): 20-6.

17. Zimmermann MB, Zeder C, Chaouki N, Saad A, Torresani T, Hurrell RF. Dual fortification of salt with iodine and microencapsulated iron: a randomized, double-blind, controlled trial in Moroccan schoolchildren. Am J Clin Nutr 2003; 77: 425-32. 